\title{
Joint Tumor Segmentation and Dense Deformable Registration of Brain MR Images
}

\author{
Sarah Parisot ${ }^{1,2,3}$, Hugues Duffau ${ }^{4}$, \\ Stéphane Chemouny ${ }^{3}$, and Nikos Paragios ${ }^{1,2}$ \\ 1 Center for Visual Computing, Ecole Centrale Paris, Chatenay Malabry, France \\ ${ }^{2}$ Equipe GALEN, INRIA Saclay - Ile de France, Orsay, France \\ ${ }^{3}$ Intrasense SAS, Montpellier, France
}

4 Département de Neurochirurgie, Hopital Gui de Chauliac, CHU Montpellier, France

\begin{abstract}
In this paper we propose a novel graph-based concurrent registration and segmentation framework. Registration is modeled with a pairwise graphical model formulation that is modular with respect to the data and regularization term. Segmentation is addressed by adopting a similar graphical model, using image-based classification techniques while producing a smooth solution. The two problems are coupled via a relaxation of the registration criterion in the presence of tumors as well as a segmentation through a registration term aiming the separation between healthy and diseased tissues. Efficient linear programming is used to solve both problems simultaneously. State of the art results demonstrate the potential of our method on a large and challenging low-grade glioma data set.
\end{abstract}

\section{Introduction}

The automatic evaluation of the evolution of brain tumors, that are often monitored through MRI imaging, is of growing interest. The methods currently used to evaluate precisely the position, size and evolution of a tumor, involve a complete 3D manual segmentation by an expert. It is, however, extremely time consuming and highly dependent on the expert, particularly in the case of the diffusively infiltrative low grade gliomas, that have fuzzy boundaries and inhomogeneous appearances [1. As it has been shown that low-grade gliomas tend to appear in preferential locations [2, the construction of statistical atlases enables the study of location dependent behaviour of tumors. As a result, both automatic segmentation and registration with a healthy atlas of tumor-bearing images are of great interest for the study of brain tumors.

State of the art segmentation methods combine efficient classification techniques 3] with low level segmentation methods [4. From such perspective, tumor detection is addressed as a classification problem where one aims at separating healthy from diseased tissues at the voxel level, while imposing smoothness

\footnotetext{
* This work was supported by ANRT (grant 147/2010), Intrasense and the European
} Research Council Starting Grant Diocles (ERC-STG-259112). 
constraints. Despite promising performance, these methods suffer from modularity and scalability. Visual and geometric appearance of tumors depend on organs, contrast agent, hardware acquisition device. The use of segmentationby-deformable registration of anatomical atlases [5] can be an answer to the aforementioned limitations due to their ability to include global consistency and deal with isolated erroneous measurements. This kind of method however suffers from the inherent link between the two problems, making the segmentation dependent on the registration quality and vice versa. A common approach to deal with the presence of tumors for registration is to mask the pathology after segmentation [6]. Another strategy is atlas seeding and simulating the tumor induced deformation in the atlas using growth models [7].

The aim of this paper is to perform tumor segmentation and registration with missing correspondences in a one shot optimization approach. Concurrent segmentation and registration is a not a new objective [89], however the problem becomes far more challenging in the presence of tumors. This is due to the fact that modeling tumor appearance and geometry is not as trivial as modeling anatomical regions, while at the same time registration is violated in the tumor area. [10] proposes registration of an atlas, deformed using a complex tumor growth model, to the patient's space. The parameters are learned using the Expectation Maximization algorithm which can be very computationally expensive and sensitive to initialization. Our approach aims at combining the modeling of both problems where the unknown variables correspond to a two layer graphical model, one that represents the $3 \mathrm{D}$ deformation field and another that refers to the $3 \mathrm{D}$ binary segmentation map. This graphical model is superimposed to the volume domain. The deformation is obtained using the discrete formulation introduced in[11]. Segmentation is obtained through a conventional graph-based formulation $[12$ where tumors and healthy tissues are separated through the use of boosting. In order to reduce complexity we adopt a reduced label grid that estimates segmentation likelihoods in the whole image domain through interpolation of the associated variables. The two layers are interconnected with a combined cost that relaxes the registration in the presence of tumors, while at the same time performs a segmentation-by-registration using the segmentation costs as criterion. Linear programming and duality 13 are used to determine the optimal solution of the combined problem. The proposed formulation is modular with respect to the registration criterion and the segmentation likelihoods, scalable since the segmentation/registration grid can be adapted to the application domain and computationally efficient. The remainder of this paper is organized as follows, section 2 describes the combined registration and segmentation method, while experimental validation is presented in the following section. Discussion and future directions conclude the paper.

\section{Graph-Based Image Registration and Segmentation}

Let us now first introduce the individual components of the method and then conclude the methodological part of the paper with their coupling. The resulting 
formulation refers to a two-layer interconnected graph where both the segmentation labels on the atlas space and the deformable registration field are determined through a one shot optimization. Such a formulation seeks both maximization of the posterior statistical likelihoods of the tumor versus healthy voxels, and optimal similarity matching between the atlas and the observed volume that is relaxed in the tumor area.

\subsection{Graph-Based Image Registration}

In the task of image registration, we seek to align a source image $A$ to a target image $I$ defined on a domain $\Omega \subset \mathbb{R}^{3}$. To this end, we seek to estimate a geometric deformation $\mathcal{T}(\mathbf{x})$ that will map A to $\mathrm{I}$.

Let us consider a sparse grid $\mathcal{G} \subset \Omega$ superimposed to the image. The deformation is evaluated on the grid's control points $\mathbf{p}$ as $\mathcal{T}(\mathbf{x})=\mathbf{x}+\sum_{p \in \mathcal{G}} \eta(\|\mathbf{x}-\mathbf{p}\|) \mathbf{d}_{\mathbf{p}}$, where $\mathbf{d}_{\mathbf{p}}$ is the displacement vector of the control point $\mathbf{p}$. The idea is to deform the source image by moving the grid's control points. The displacement of a voxel $\mathbf{x}$ of the image will be determined by the control point's displacements and the influence of each control point on $\mathbf{x}$, which is given by the projection function $\eta$. A typical example of projection function would be cubic B-splines. The optimal deformation field should minimize the distance between the target image $I(\mathbf{x})$ and deformed image $A(\mathcal{T}(\mathbf{x}))$, that is evaluated by a similarity function $\rho$ :

$$
E(\mathcal{T})=\frac{1}{|\mathcal{G}|} \sum_{\mathbf{p} \in \mathcal{G}} \int_{\Omega} \bar{\eta}(\|\mathbf{x}-p\|) \rho(I(x), A(\mathcal{T}(\mathbf{x}))) d x
$$

The similarity function being defined on the image domain $\Omega$, a function $\bar{\eta}$ is introduced to back project the voxel wise information on the grid's control points. It determines how much voxel $\mathbf{x}$ influences the control point $\mathbf{p}$.

We adopt the discrete approach described in [11, in which problem (1) is reformulated as a labeling problem. To this end, we define a set of labels $\mathcal{L}=$ $\left\{l^{1}, \ldots, l^{k}\right\}$ and a discrete displacement set $\Delta=\left\{\mathbf{d}^{\mathbf{1}}, \ldots, \mathbf{d}^{\mathbf{k}}\right\}$. Each label corresponds to a specific displacement vector so that assigning a label $l_{p} \in \mathcal{L}$ to a control point $\mathbf{p}$ imposes the corresponding displacement $\mathbf{d}^{\mathbf{i}}$ of the node. The deformation field can be reformulated as: $\mathcal{T}_{l}(\mathbf{x})=\mathbf{x}+\sum_{p \in \mathcal{G}} \eta(\|\mathbf{x}-\mathbf{p}\|) \mathbf{d}^{\mathbf{l}} \mathbf{p}$. This enables to pose the problem as a discrete Markov Random Field (MRF) optimization with respect to the labeling $l$, where the goal is to minimize the following energy:

$$
E_{\text {def }}(l)=\frac{1}{|\mathcal{G}|} \sum_{p \in \mathcal{G}} V_{p}\left(l_{p}\right)+\lambda \sum_{p \in \mathcal{G}} \sum_{q \in \mathcal{N}(p)} V_{p q}\left(l_{p}, l_{q}\right)
$$

$V_{p}\left(l_{p}\right)$ can be approximated as $\left.V_{p}\left(l_{p}\right) \approx \int_{\Omega} \bar{\eta}(\|\mathbf{x}-\mathbf{p}\|) \rho\left(I(x), A\left(\mathbf{x}+\mathbf{d}^{\mathbf{l}_{\mathbf{p}}}\right)\right)\right) d x$ $V_{p q}$ is a pairwise constraint that imposes local smoothness of the deformation field and $\lambda$ is a constant parameter balancing the single and pairwise costs. This formulation is not sufficient in the case of lacking correspondences between the images. In these areas, the source image will be heavily deformed and information will be lost. 


\subsection{Graph-Based Image Segmentation}

In order to automatically segment tumor voxels, we seek to construct a classifier that will describe the likelihood that each voxel belongs to a tumor. One simple and efficient way to do so is to learn the classifier based on a vector of image features and boosting. We use the Gentle Adaboost algorithm [3], that is known to be more robust to noise and outliers than other boosting algorithms. We adopt three feature spaces. First, we rely on the intensity values in the voxel of interest $\mathbf{x}_{\mathbf{i}}$ and its neighborhood. We include in the feature vector an intensity patch centered on $\mathbf{x}_{\mathbf{i}}$, the median, entropy and standard deviation of patches of variable sizes. Second, we use Gabor features [14] on 2 scales and 10 orientations. Last, the tumor's presence will introduce some lack of symmetry between the hemispheres. Assuming we know an approximate symmetry plane $\Pi$, we compute a symmetry feature as $\operatorname{Sym}\left(\mathbf{x}_{\mathbf{i}}\right)=\frac{1}{k} \sum_{\mathcal{N}\left(\mathbf{x}_{\mathbf{i}}\right)} I\left(\mathbf{x}_{\mathbf{i}}\right)-\frac{1}{k} \sum_{\mathcal{N}\left(\mathbf{x}_{\mathbf{i}}\right)} I\left(\mathbf{x}_{\mathbf{i} \Pi}\right), \mathbf{x}_{\mathbf{i} \Pi}$ being the symmetric of $\mathbf{x}_{\mathbf{i}}$ with respect to $\Pi$, and $\mathcal{N}$ a neighborhood around $\mathbf{x}_{\mathbf{i}}$ introduced to reduce the approximation error. The classifier's output can be converted into a tumor $p_{t m}(\mathbf{x})$ and a background probability $p_{b g}(\mathbf{x})$. Both probabilities are fixed after training.

The problem of tumor segmentation can be cast as an MRF optimization on the whole volume $I$, where each voxel gets a label $l \in\{0,1\}$ identifying it as tumor or background:

$$
E_{\text {seg }}(l)=\sum_{\mathbf{x}} V_{x}(l(\mathbf{x}))+\lambda_{\text {seg }} \sum_{\mathbf{x}} \sum_{\mathbf{y} \in \mathcal{N}(\mathbf{x})} V_{x y}(l(\mathbf{x}), l(\mathbf{y}))
$$

The single costs impose a labeling according to the classification likelihoods: $V_{x}(l(\mathbf{x}))=-\log \left(p_{b g}(\mathbf{x})\right)(1-l((\mathbf{x})))-\log \left(p_{t m}(\mathbf{x})\right) l((\mathbf{x})) . V_{x y}$ is a pairwise cost added to impose local consistency of the segmentation and $\lambda_{\text {seg }}$ balances the single and pairwise costs. The segmentation task could also benefit from the registration, since existing correspondences between the atlas and the image to be segmented could eliminate false detections of the classifier.

\subsection{Combined Registration and Segmentation}

Let us now describe the main contribution of this paper: the joint segmentationregistration framework. Let us consider that the source image $A$, a healthy brain image, is to be registered to a target brain image $I$ featuring a low-grade glioma. In the method presented in section 2.1. the tumor's presence leads to heavy deformation of the brain and loss of structural information. The aim of our method is to amend this problem by detecting the tumor's position and not taking it into account during registration, while at the same time obtaining a precise segmentation of the tumor.

We seek to evaluate the geometric deformation $\mathcal{T}(\mathbf{x})$ and the tumor's position $\mathcal{S}(\mathbf{x})$ through the same optimization. We adopt a discrete MRF formulation in which each grid node $\mathbf{p}$ is assigned a label $l \in \mathcal{L}=\left\{l^{1}, \ldots, l^{2 k}\right\}$. Each label corresponds to a pair $\{s, \mathbf{d}\} \in\{0,1\} \times\left\{\mathbf{d}^{\mathbf{1}}, \ldots, \mathbf{d}^{\mathbf{k}}\right\}$. The first term $s^{l_{p}}$ will simply 
characterize the node as tumor or background, while the second one $\mathbf{d}^{\mathbf{l}} \mathbf{p}$ determines the node's displacement. The segmentation and displacement information is propagated to the whole image by cubic B-spline interpolation:

$$
\mathcal{T}(\mathbf{x})=\mathbf{x}+\sum_{p \in \mathcal{G}} \eta(\|\mathbf{x}-p\|) \mathbf{d}^{\mathbf{l}_{\mathbf{p}}} \quad \text { and } \quad \mathcal{S}(\mathbf{x})=H\left(\sum_{p \in \mathcal{G}} \eta(\|\mathbf{x}-p\|) s^{l_{p}}-0.5\right)
$$

Due to the interpolation, the segmentation map $\mathcal{S}(\mathbf{x})$ is not binary. We deal with this issue by thresholding the map using the heaviside function $\mathrm{H}$.

Let us now proceed with the definition of the singleton cost $V_{p}\left(l_{p}\right)$. In the case of healthy voxels, the evaluation of the deformation is still based on a similarity measure between the source and target image. In the tumor area $\left(s^{l_{p}}=1\right)$, we cannot rely on the similarity measure and therefore introduce a constant cost $C_{t m}$ as suggested in [15] for stereo matching:

$$
V_{d e f}\left(l_{p}\right)=\int_{\Omega} \bar{\eta}(\|\mathbf{x}-\mathbf{p}\|)\left(\left(1-s^{l_{p}}\right) \rho\left(V(\mathbf{x}), A\left(\mathbf{x}+\mathbf{d}^{\mathbf{l}_{\mathbf{p}}}\right)\right)+s^{l_{p}} C_{t m}\right) d x
$$

This potential enables registration without taking into account the area detected as tumor (highly dissimilar points in the image). While part of the tumor can be detected using this single potential, the lack of similarity alone is not sufficient to efficiently segment the tumor. Only tumors voxels with high contrast enhancement will be detected. Furthermore, dissimilarity doesn't necessarily correspond to a tumor. To enhance the segmentation and prevent false positives, we make use of the classifier built in section 2.1. We couple the deformation potential with a second one acting mainly on the segmentation space:

$$
V_{s e g}\left(l_{p}\right)=\int_{\Omega} \bar{\eta}(\|\mathbf{x}-\mathbf{p}\|)\left(-\log \left(p_{b g}\left(\mathbf{x}+\mathbf{d}^{\mathbf{l}_{\mathbf{p}}}\right)\right)\left(1-s^{l_{p}}\right)-\log \left(p_{t m}\left(\mathbf{x}+\mathbf{d}^{\mathbf{l}_{\mathbf{p}}}\right)\right) s^{l_{p}}\right) d x
$$

This potential imposes that voxels with a high probability of being tumor $\left(p_{t m}\right)$ are labeled accordingly. It takes the deformation into account since the classifier's score is defined on the target image: the better the source image is aligned to the target image, the more precise becomes the tumor's position. We can now define the singleton cost as $V_{p}\left(l_{p}\right) \approx \alpha V_{d e f}\left(l_{p}\right)+(1-\alpha) V_{\text {seg }}\left(l_{p}\right)$, where $\alpha$ is a constant coefficient of key importance balancing the 2 potentials' influence. If $\alpha$ is high, the registration will be prioritized, resulting in a good registration (except in the tumor area) and poor segmentation. On the contrary, imposing a low $\alpha$ yields a good segmentation (assuming the classifier is efficient), while the registration quality will be deteriorated. As for the pairwise cost, it is set as:

$$
V_{p q}\left(l_{p}, l_{q}\right)=\alpha \lambda \frac{\left\|\mathbf{d}^{\mathbf{l}_{\mathbf{p}}}-\mathbf{d}^{\mathbf{l}_{\mathbf{q}}}\right\|}{\|\mathbf{p}-\mathbf{q}\|}+(1-\alpha) \frac{\left|s^{l_{p}}-s^{l_{q}}\right|}{\|\mathbf{p}-\mathbf{q}\|}
$$

In order to recover the optimal labeling solution, we use Fast-PD[13], an efficient MRF optimization method based on linear programming. 


\section{Experimental Validation}

We adopt a multi-scale incremental approach (both on the image and grid resolutions) were the tumor presence will have an increasing impact on the process. We evaluated the deformation and segmentation on 3 image levels and 4 grid levels $(9 \times 9 \times 5$ to $65 \times 65 \times 37)$. The parameter $C_{t m}$ was increased at each level, from 5 to 10 times the mean value of the similarity measure, while the parameter $\alpha$ is progressively reduced from 1 to $0.015 . \lambda$ and $\lambda_{\text {seg }}$ were set at 20 and 3 respectively. At each level $t$, new deformation and segmentation fields are estimated w.r.t. the source image deformed in level $t-1$. The deformation field $\mathcal{T}^{t}(\mathbf{x})$ is updated by composition with the one obtained at level $t-1$. Since false positives are less likely to appear in the coarse levels, we add a penalty term as $V_{\text {pen }}^{t}\left(\mathbf{l}_{\mathbf{p}}\right)=-\int_{\Omega} \bar{\eta}(\|\mathbf{x}-\mathbf{p}\|)\left(1-s^{l_{p}}\right) \exp (-t) d x$ to voxels labeled as background $\left(S^{t-1}(\mathbf{x})=0\right)$. We use the Sum of Absolute Differences as similarity criterion.

The healthy brain template used for registration was a 3D MRI FLAIR image, of size $256 \times 256 \times 24$, and resolution $0.9 \times 0.9 \times 5.45 \mathrm{~mm}^{3}$. All images in our data-set (93 images) were 3D FLAIR images of different patients with low-grade gliomas that have been manually segmented by an expert. Their sizes ranged from $256 \times 256 \times 24$ to $512 \times 512 \times 33$, and resolution from $0.4 \times 0.4$ to. $9 \times 0.9 \mathrm{~mm}^{2}$ in the $(\mathrm{x}, \mathrm{y})$ plane and 5.3 to $6.4 \mathrm{~mm}$ in the $\mathrm{z}$ plane. The smallest tumor was $3.5 \mathrm{~cm}^{3}$, and the largest was $230 \mathrm{~cm}^{3}$. Preprocessing involved skull stripping and intensity regularization. In order to avoid losing tumor vs healthy tissue contrast, we simply set all images to the same median and interquartile range as the reference pose, without taking into account background voxels. Eventually, all images were rigidly registered to the reference pose, which permitted to use the template's symmetry plane to evaluate the symmetry feature for the boosting classifier. 36 randomly selected volumes were used for boosting learning. The patches sizes for intensity statistics were $k \times k \times 3$, with $k=\{3,5,7\}$.

We tested our framework on the 57 remaining images. Overall computational time was approximately 6 minutes. The registration was compared qualitatively to individual registration where the pathology is masked using the manual segmentation masks (enabling comparison to a sequential approach). Some visual registration results, along with the corresponding deformation maps are shown in Fig. 2. We observe high visual correspondences between the target and registered images outside the tumor area for both methods. As shown by the individual registration's deformation maps, deformations inside the tumor area are very important and excessively unnatural. Using our method, the deformations are much smoother and the deformed image's anatomy is respected, showing that our method performs significantly better in the tumor area. The automatic segmentation (A) was evaluated w.r.t the manual segmentation (M) using the Dice coefficient, the rate of false positives $\left(F P=\frac{\|A\|-\|M \cap A\|}{\|A\|}\right)$ and the Mean Absolute Distance (MAD) between contours. Results were compared to the voxel wise individual segmentations. Comparative boxplots are shown in Fig. 1]. The median dice increases from $77 \%$ to $80 \%$, while false positives significantly diminished (median $30 \%$ to $20 \%$ ) and MAD values also diminished. 


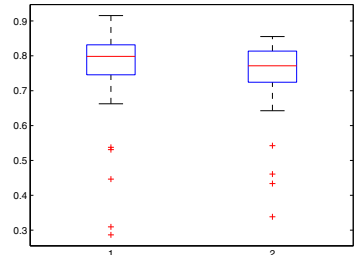

(a)

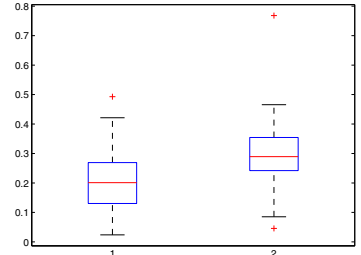

(b)

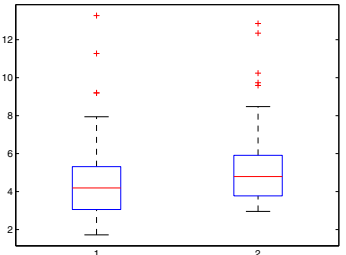

(c)

Fig. 1. Boxplots of the (a) Dice values, (b) false positive rates and MAD (c) for the joint framework (1) and the individual segmentation method (2)
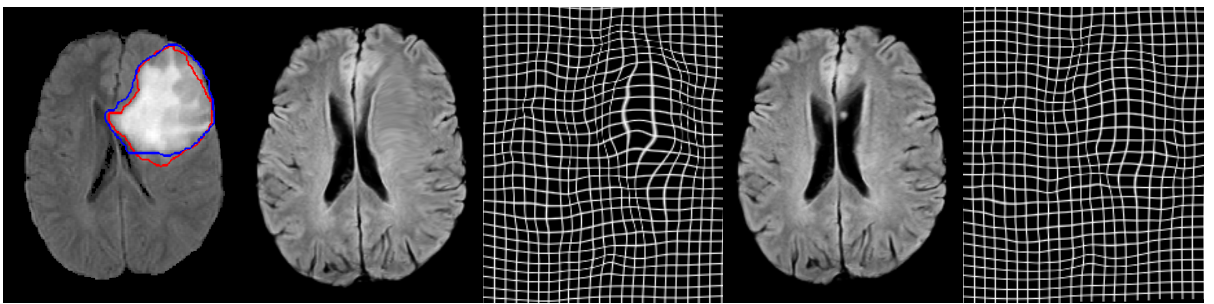

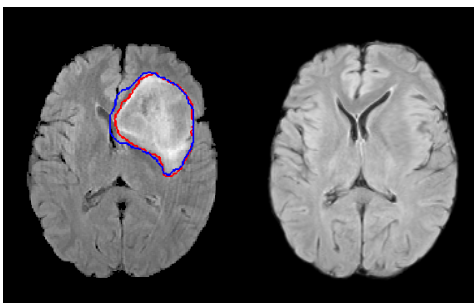

(a)

(b)

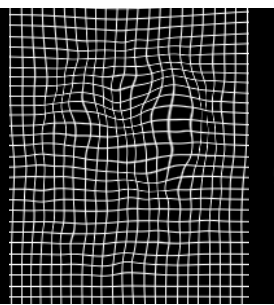

(c)

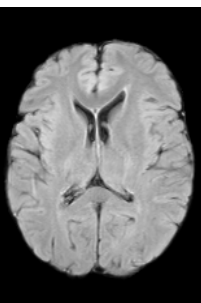

(d)

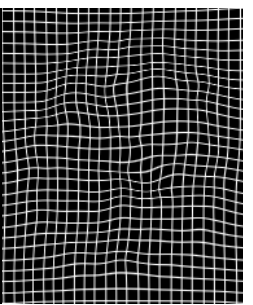

(e)

Fig. 2. Visual registration and segmentation results. (a) Segmented target image (manual (red) and automatic (blue) segmentations), (b) Individual registration, (d) our framework, (c,e) associated deformation fields.

\section{Discussion}

In this paper we have proposed a novel, efficient and principled method for combined tumor segmentation and dense registration. This was achieved through a two-layer interconnected graphical model that was optimized using a single shot approach towards optimal recovery of both variable spaces. Extensive validation concerning the case of low-grade gliomas was considered to evaluate the performance of the method, leading to very promising results. Introducing prior knowledge in the reference space as suggested in [2] is a straightforward extension of the proposed formulation which could further improve the performance of the method while allowing the characterization of tumors. Another possible extension of the method is the use of recent advances in MRF learning [16] towards 
encoding prior knowledge using spatially varying coefficients of the corresponding segmentation framework that could be learned from training examples.

\section{References}

1. Kaus, M., Warfield, S., Nabavi, A., Black, P., Jolesz, F., Kikinis, R.: Automated Segmentation of MR Images of Brain Tumors. Radiology 218(2), 586-591 (2001)

2. Parisot, S., Duffau, H., Chemouny, S., Paragios, N.: Graph Based Spatial Position Mapping of Low-Grade Gliomas. In: Fichtinger, G., Martel, A., Peters, T. (eds.) MICCAI 2011, Part II. LNCS, vol. 6892, pp. 508-515. Springer, Heidelberg (2011)

3. Friedman, J., Hastie, T., Tibshirani, R.: Additive Logistic Regression: a Statistical View of Boosting. The Annals of Statistics 38(2), 337-407 (2000)

4. Lee, C., Wang, S., Murtha, A., Brown, M., Greiner, R.: Segmenting Brain Tumors Using Pseudo-Conditional Random Fields. In: Metaxas, D., Axel, L., Fichtinger, G., Székely, G. (eds.) MICCAI 2008, Part I. LNCS, vol. 5241, pp. 359-366. Springer, Heidelberg (2008)

5. Prastawa, M., Bullit, E., Moon, N., Leemput, K., Gerig, G.: Automatic Brain Tumor Segmentation by Subject Specific Modification of Atlas Priors. Academic Radiology 10(12), 1341-1348 (2003)

6. Stefanescu, R., Commowick, O., Malandain, G., Bondiau, P., Ayache, N., Pennec, $\mathrm{X}$.: Non-rigid atlas to subject registration with pathologies for conformal brain radiotherapy. Springer (2004)

7. Bach Cuadra, M., De Craene, M., Duay, V., Macq, B., Pollo, C., Thiran, J.: Dense deformation field estimation for atlas-based segmentation of pathological MR brain images. Computer Methods and Programs in Biomedicine 84(2-3), 66-75 (2006)

8. Yezzi, A., Zollei, L., Kapur, T.: A variational framework for integrating segmentation and registration through active contours. Medical Image Analysis 7, 171-185 (2003)

9. Wyatt, P.P., Noble, J.A.: MAP MRF joint segmentation and registration of medical images. Medical Image Analysis 7(4), 539-552 (2003)

10. Gooya, A., Pohl, K.M., Bilello, M., Biros, G., Davatzikos, C.: Joint Segmentation and Deformable Registration of Brain Scans Guided by a Tumor Growth Model. In: Fichtinger, G., Martel, A., Peters, T. (eds.) MICCAI 2011, Part II. LNCS, vol. 6892, pp. 532-540. Springer, Heidelberg (2011)

11. Glocker, B., Komodakis, N., Tziritas, G., Navab, N., Paragios, N.: Dense image registration through MRFs and efficient linear programming. Medical Image Analysis 12(6), 731-741 (2008)

12. Boykov, Y., Funka-Lea, G.: Graph Cuts and Efficient ND Image Segmentation. International Journal of Computer Vision 70(2), 109-131 (2006)

13. Komodakis, N., Tziritas, G., Paragios, N.: Performance vs computational efficiency for optimizing single and dynamic MRFs: Setting the state of the art with primaldual strategies. Computer Vision and Image Understanding 112(1), 14-29 (2008)

14. Michel, F., Bronstein, M., Bronstein, A., Paragios, N.: Boosted metric learning for 3D multi-modal deformable registration. In: ISBI, pp. 1209-1214. IEEE (2011)

15. Kang, S., Szeliski, R., Chai, J.: Handling occlusions in dense multi-view stereo. In: CVPR, vol. 1, pp. I-103. IEEE (2001)

16. Komodakis, N.: Efficient training for pairwise or higher order CRFs via dual decomposition. In: CVPR, pp. 1841-1848 (2011) 